\title{
Mechanisms of cough provocation and cough resolution in neonates with bronchopulmonary dysplasia
}

\author{
Sudarshan R. Jadcherla ${ }^{1-3}$, Kathryn A. Hasenstab ${ }^{1,2}$, Reza Shaker ${ }^{4}$ and Robert G. Castile ${ }^{1,2,5}$
}

BACKGROUND: Cough and deglutition are protective mechanisms that defend against aspiration. We identified mechanisms associated with cough provocation as well as those associated with cough resolution in infants with bronchopulmonary dysplasia (BPD).

METHODS: Manometry signatures of cough were recognized in 16 premature infants with BPD undergoing concurrent esophageal manometry, respiratory inductance plethysmography, and nasal air flow measurements. Pretussive and posttussive pharyngo-esophageal motility changes were analyzed. Mechanisms associated with cough and mechanisms that restored respiratory and esophageal normalcy were analyzed. RESULTS: We analyzed 312 cough events during 88 cough clusters; $97 \%$ were associated with recognizable manometric patterns. Initial mechanisms related with coughing included nonpropagating swallow (59\%), upper esophageal sphincter (UES) reflex contraction (18\%), and lower esophageal sphincter (LES) relaxation (14\%). UES and LES dysfunction was present in 69\% of nonpropagating swallow-associated cough clusters. Mechanisms restoring post-tussive normalcy included primary peristalsis (84\%), secondary peristalsis (8\%), and none recognized (8\%). UES contraction reflex was associated with cough clusters more frequently in infants on nasal continuous positive airway pressure (NCPAP) $(\mathrm{OR}=9.13,95 \% \mathrm{Cl}=1.88-44.24)$. CONCLUSION: Cough clusters in infants with BPD had identifiable etiologies associated with esophageal events; common initial mechanisms were of upper aerodigestive origin, while common clearing mechanisms were peristaltic reflexes.

$\mathbf{T}$ he cough reflex, defined as a forceful exhalation preceded by glottal closure, is an important aerodigestive protective mechanism permitting clearance of secretions and foreign material from the airway, thus abrogating aspiration $(1,2)$. Cough can also be a marker for airway hyper-reactivity. Coughing can cause irritability, pain, sleep disturbances, fatigue, hoarseness, and bronchospasm. In adults and children, cough is frequently related to respiratory tract infection, asthma, or gastroesophageal reflux disease (GERD) (3-5). Cough has been reported to occur 21 times per day (1-171 times per day, range) in infants with bronchopulmonary dysplasia (BPD) (6) and may occur with other aerodigestive symptoms such as dysphagia, stridor, wheezing, grunting, costal retraction, nasal flaring, and/or tachypnea.

The incidence of BPD, defined as oxygen requirement beyond $36 \mathrm{wk}$ postmenstrual age (7), remains high in verylow-birth-weight preterm infants with gestational ages ranging from 22 to $32 \mathrm{wk}$, ranging from 30 to $54 \%$ (8-10). Average length of initial hospitalization is about $94 \mathrm{~d}$, with an average cost of $\$ 103,151$ in the United States (11). These infants frequently experience feeding difficulties due to abnormal coordination of respiration and swallowing, poor oromotor skills, swallowing dysfunction, and/or GERD (12-14). Resultantly, $\sim 23 \%$ of patients undergo gastrostomy tube placement in the first 2 y of life (15).

Prolonged coughing episodes can alter physiological status. Cough also plays a role in protecting against aspiration. What causes and resolves cough episodes in infants with BPD is not well understood. Therefore, in infants with BPD and feeding difficulties, we hypothesized that the cough reflex during manometric evaluation is associated with aerodigestive events specifically swallowing and/or gastroesophageal reflux. Our objectives were to define aerodigestive mechanisms that (i) are associated with the cough reflex and (ii) restore aerodigestive normalcy following coughing episodes. We investigated these objectives using basal and provocative pharyngo-esophageal manometry while concurrently monitoring thoracic and abdominal respiratory effort and rhythms.

\section{RESULTS}

Demographic, Disease, and Discharge Outcome Characteristics

Data presented as median (range) from 16 infants (10 male) prematurely born at $25 \mathrm{wk}$ (24-34) gestation, suffering from BPD, feeding difficulties and symptoms of cough were examined during esophageal manometry. Birth weights were 0.8 (0.6-2.1) kg birth weight with 1 infant in the low-birth-weight category $(<2.5 \mathrm{~kg}), 1$ infant in the very-low-birth-weight category $(<1.5 \mathrm{~kg})$, and the remaining 14 in the extremely lowbirth-weight category $(<1.0 \mathrm{~kg})$. Duration of ventilation was $84(6-112) \mathrm{d}$ and duration of subsequent continuous positive

'The Neonatal and Infant Feeding Disorders Program, Center for Perinatal Research, Nationwide Children's Hospital Research Institute, Columbus, Ohio; ${ }^{2}$ The Neonatal Aerodigestive Pulmonary Program, Nationwide Children's Hospital, Columbus, Ohio; ${ }^{3}$ Divisions of Neonatology, Pediatric Gastroenterology and Nutrition, Department of Pediatrics, The Ohio State University College of Medicine, Columbus, Ohio; ${ }^{4}$ Gastroenterology and Internal Medicine, Medical College of Wisconsin, Milwaukee, Wisconsin; ${ }^{5}$ Division of Respiratory Medicine, Department of Pediatrics, The Ohio State University College of Medicine, Columbus, Ohio. Correspondence: Sudarshan R. Jadcherla (Sudarshan.Jadcherla@nationwidechildrens.org) 
airway pressure (CPAP) was $29(0-95)$ d. Length of hospital stay was 189 (70-673) d. One subject died due to progression of BPD and persistent pulmonary hypertension. Nine infants received a gastrostomy tube. Six subjects had a neurologic comorbidity. Three subjects had surgical ligation of a patent ductus arteriosus.

Clinical characteristics at esophageal manometric evaluation were as follows: (i) postmenstrual age 44.1 (37.0-56.3) wk, weight $3.6(2.1-5.5) \mathrm{kg}$, (ii) mode of respiratory support, i.e., nasal continuous positive airway pressure: nasal cannula: room air were 50: 44: 6\% respectively, and (iii) feeding method, i.e., exclusive gavage: gavage and oral: exclusive oral were 50: 44: $6 \%$ respectively. At discharge: (i) $87 \%$ of patients were breathing room air while $13 \%$ were on supplemental oxygen via nasal cannula (ii) feeding method, i.e., exclusive gastrostomy tube: gastrostomy tube and oral: oral were 7: 53: 40\% respectively.

During a total of $24.9 \mathrm{~h}$ of cumulative manometry data, 312 individual cough events and 88 cough clusters were recognized and analyzed in detail. Median (range) number of cough clusters per subject were 5.5 (3-9) and cough events per cough cluster were 3 (1-15). Of the 88 cough clusters, $97 \%$ had recognizable aerodigestive mechanisms primarily associated with cough; of which $68 \%$ occurred spontaneously and $32 \%$ occurred with stimulus. Characteristics of pharyngo-esophageal motility before, during, and after cough clusters are shown (Table 1). The durations of respiratory rhythm disturbance during spontaneous cough clusters and stimulus related cough clusters were $22.6 \pm 1.5$ and $25.8 \pm 2.5$ s, respectively.

\section{Aerodigestive Mechanisms Associated With Cough Clusters}

Overall, cough origin and initial mechanisms associated with cough are displayed in (Figure 1a) and (Figure 1b) respectively. For both spontaneous and stimulus-related cough clusters $(n=$ 88 ), the majority of initial mechanisms associated with cough originated in the upper aerodigestive tract 68 (77\%). Frequent initial mechanisms associated with cough clusters in the upper aerodigestive tract was nonpropagating swallowing in 52 (59\%), and in the lower aerodigestive tract lower esophageal sphincter (LES) relaxation in 12 (14\%) (Figure 1b). Gastroesophageal reflux (GER) mechanisms (LES relaxation with retrograde common cavity esophageal pressure change) were associated in only 17 (20\%) of cough clusters (Figure 1b). When nonpropagating swallow occurred as the initial mechanism associated with cough clusters $(N=52)$, we observed the following signs: (i) nonpropagating swallow occurred during inspiration in $24(46.1 \%)$ of clusters; (ii) upper esophageal sphincter (UES) exhibited incomplete relaxation in $36(69.2 \%)$ of clusters; (iii) LES also exhibited dysfunction in 36 (69.2\%) of clusters. Types of LES dysfunction

Table 1. Manometric characteristics of cough in infants with BPD

\begin{tabular}{|c|c|c|c|c|c|c|}
\hline $\begin{array}{l}\text { Characteristic } \\
(\mathrm{mmHg})\end{array}$ & $\begin{array}{l}\text { Baseline } \\
\text { precough }\end{array}$ & Cough onset & Peak & Cough offset & $\begin{array}{l}\text { Baseline } \\
\text { postcough }\end{array}$ & $\begin{array}{l}\text { Kruskal-Wallis } \\
P \text { value }\end{array}$ \\
\hline Upper esophageal sphincter & $19.5(2-99)^{\dagger}$ & $32.5(-9-201)^{*,+}$ & $90(15-224)^{*}$ & $41(-4-134)^{*, \dagger}$ & $22(6-90)^{\dagger}$ & $<0.001$ \\
\hline Lower esophageal sphincter & $23.5(-9-106)^{\dagger}$ & $14.5(-43-95)^{*,+}$ & $104.5(56-203)^{*}$ & $15(-25-124)^{\dagger}$ & $24(-6-81)^{\dagger}$ & $<0.001$ \\
\hline Gastric & $10(0-63)^{+}$ & $15.5(3-71)^{*,+}$ & $85(31-176)^{*}$ & $14(3-51)^{*,+}$ & $10(2-33)^{+}$ & $<0.001$ \\
\hline
\end{tabular}

Values are presented as median (range), ${ }^{*} P<0.05 \mathrm{vs}$. precough baseline, ${ }^{\dagger} P<0.05 \mathrm{vs}$. peak.

a

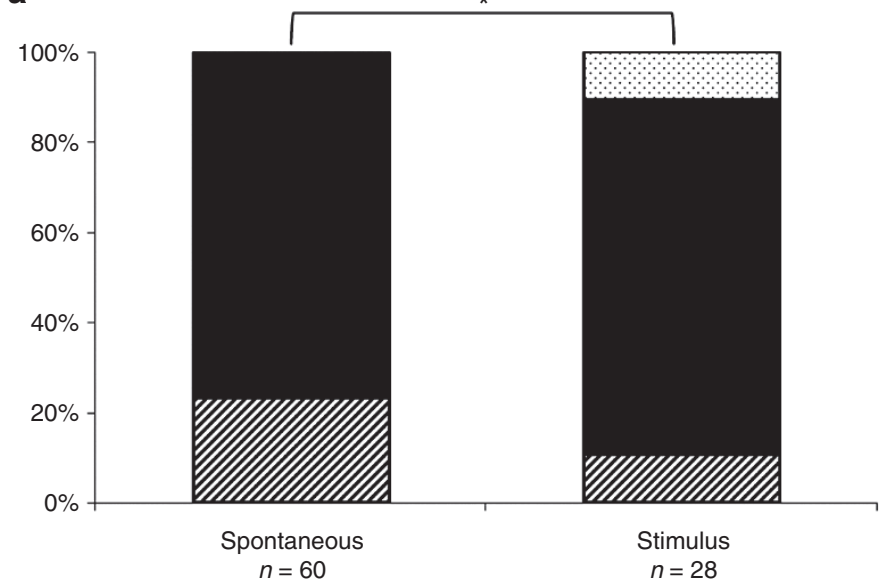

b

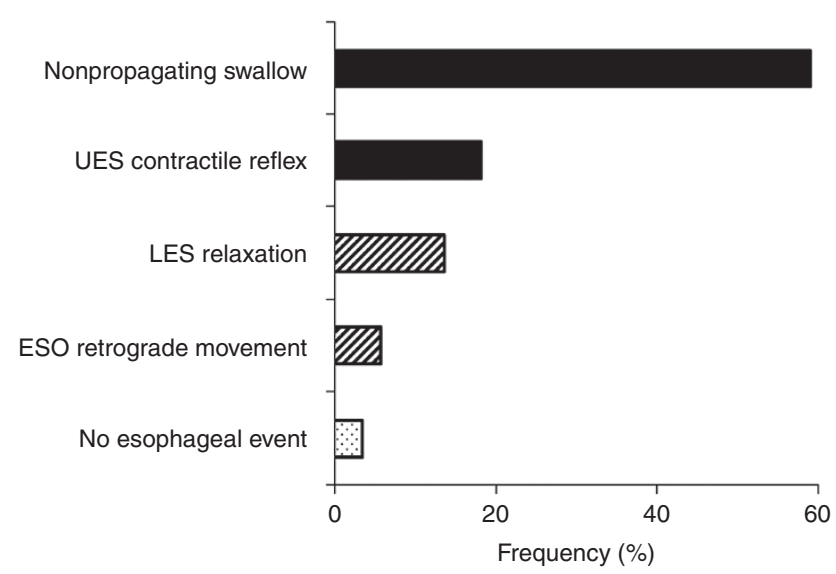

Figure 1. Initial aerodigestive mechanisms associated with cough clusters: $\square$ Upper aerodigestive tract (nonpropagating swallow, upper esophageal sphincter (UES) contractile reflex), $\mathbb{A}$ Lower aerodigestive tract (lower esophageal sphincter (LES) relaxation, esophageal retrograde movement), 鮦 Airway (no esophageal event associated with cough cluster), (a) Location of origin (Upper aerodigestive tract: Lower aerodigestive tract, Airway, $\%)$ for spontaneous $(n=60)$ and stimulus $(n=28)$ related cough clusters were 77: 23: 0 vs. $78: 11: 11$ respectively, ${ }^{*} P=0.03$. (b) Initial aerodigestive mechanism associated with cough: Nonpropagating swallow 52 (59\%) and UES contractile reflexes 16 (18\%) were the most frequent aerodigestive mechanisms associated with cough which originate in the upper aerodigestive tract. Other less common initial mechanisms associated were LES relaxation $12(14 \%)$, esophageal retrograde movement $5(6 \%)$, and no esophageal events associated 3 (3\%). 
a

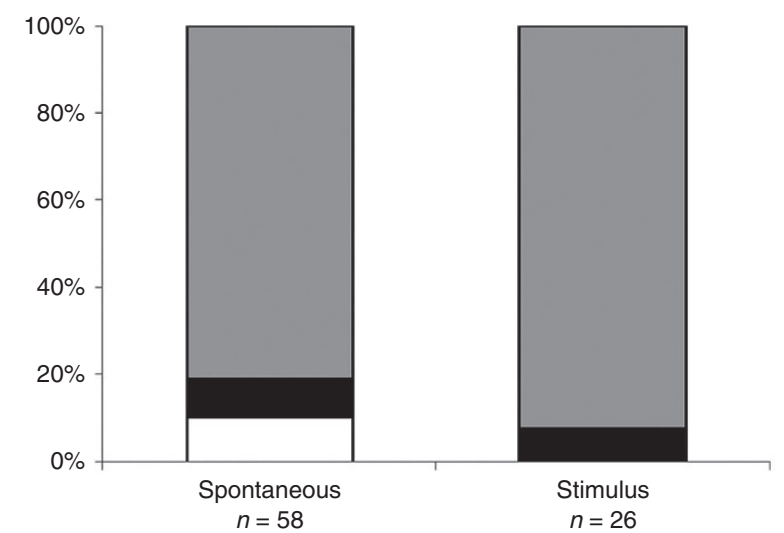

b

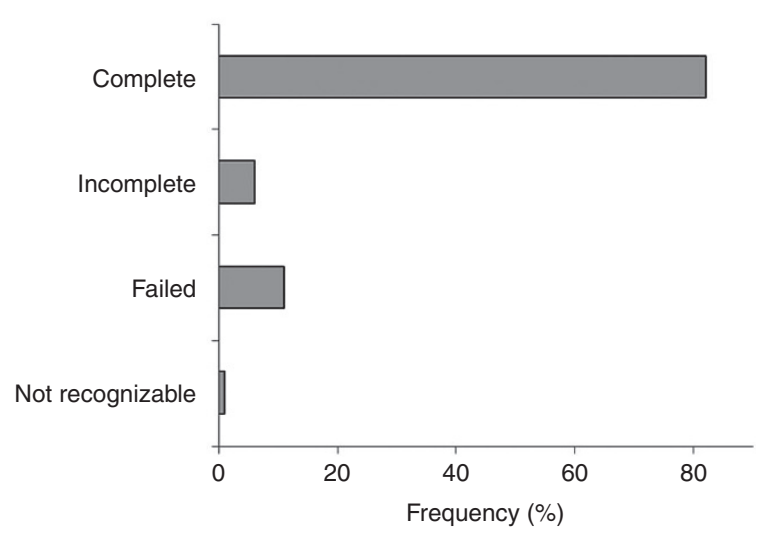

Figure 2. Post-tussive clearing mechanisms associated with cough clusters: $\square$ Primary peristalsis, $\square$ Secondary peristalsis, $\square$ None. (a) Clearance mechanism type (Primary peristalsis: secondary peristalsis: none, \%) for spontaneous $(n=60)$ and stimulus $(n=28)$ related cough clusters were $81: 9: 10$ vs. 92 : 0: 8 respectively. Primary peristalsis was the dominant clearance mechanism for respiratory and esophageal recovery. (b) Primary peristalsis clearance mechanism propagation: Complete propagation was the dominant facilitator of clearance in $58(82 \%)$, followed by incomplete propagation $4(6 \%)$, failed propagation $8(11 \%)$, and not recognizable $1(1 \%)$.

recognized were: absence of relaxation $5(13.9 \%)$, presence of sustained contraction $4(11.1 \%)$, prolonged relaxation $6(16.7 \%)$, or incomplete relaxation 21 (58.3\%).

\section{Categorization of Initial Aerodigestive Mechanisms Subcategorized by Spontaneous Cough Clusters and Stimulus-Induced Cough Clusters}

Initial aerodigestive mechanisms associated with cough for spontaneous cough clusters $(n=60)$ were: nonpropagating swallow 39 (65.0\%), UES contractile reflex 7 (11.7\%), LES relaxation 10 (16.7\%), and esophageal retrograde movement 4 (6.7\%). During stimulus induced cough clusters $(n=28)$, initial mechanisms associated were: nonpropagating swallow 13 (46.4\%), UES contractile reflex $9(32.1 \%)$, LES relaxation $2(7.1 \%)$, esophageal retrograde movement in 1 (3.6\%), and none detected in $3(10.7 \%)$.

\section{Recognition of Multiple Physiological Patterns During Cough Clusters}

Subsequent aerodigestive reflex mechanisms were observed following the initial reflex in $76(86.4 \%)$ of the 88 cough clusters. More than one mechanism occurred during this physiological reflex patterns; for example, during these 76 cough clusters, nonpropagating swallow occurred 14 times (18.4\%), deglutition during inspiration occurred 28 times (36.8\%), UES contractile reflex occurred 38 times (50.0\%), LES relaxation reflex occurred 31 times (40.8\%), and/or esophageal retrograde peristaltic movement during 24 cough clusters (31.6\%).

\section{Post-tussive Peristaltic Mechanisms Restoring Aerodigestive Normalcy}

Post tussive mechanisms restoring esophageal and respiratory normalcy are displayed (Figure 2). Frequency of peristaltic propagation, i.e., complete: incomplete: failed: nonrecognizable patterns, were $81: 8: 11$ : $0 \%$ respectively for spontaneous cough clusters, and 77: 8: 11: $4 \%$ respectively for stimulus related cough clusters.

\section{Comparing Cough Initiating and Ameliorating Mechanisms in} Infants on NCPAP $(n=8)$ vs. Non-NCPAP $(n=8)$

Increased frequency of UES contractile reflex as the initial mechanism associated with cough was noted in nasal continuous positive airway pressure (NCPAP) vs. non-NCPAP, 28.6 vs. $5.1 \%$ respectively $(\mathrm{OR}=9.13,95 \% \mathrm{CI}=1.88-44.24)$. Cough ameliorating mechanisms (primary peristalsis: secondary peristalsis: none) were similar in the NCPAP vs. non-NCPAP groups, $82: 5: 13 \%$ vs. $86: 11: 3 \%$, respectively, and mechanisms of peristaltic propagation, i.e., complete: incomplete: failed, were also similar between the two subgroups, 26: 40: $34 \%$ vs. 28: $47: 25 \%$ respectively.

\section{DISCUSSION}

In infants with BPD demonstrating coughing during manometric testing, the dominant initial mechanisms associated with cough were non-propagating swallowing and UES contractile reflexes. Thus, cough events frequently had upper aerodigestive origins. When nonpropagating swallowing occurred as the initial mechanism, UES and LES reflex abnormalities were noted and may have contributed to ineffective esophageal clearance. Furthermore, GER was an initial mechanism in only $20 \%$ of cough clusters. Completely propagated primary peristalsis was the dominant esophageal clearance mechanism. The durations of respiratory rhythm disturbances during cough clusters were also of considerable length.

Numerous studies have evaluated esophageal motility and cough in adults (16-19), from which multiple comparisons can be made: (i) Baseline esophageal motility characteristics of the UES, middle esophagus, LES, and stomach of our population closely resembles those reported in the elderly population (19). UES protection has also been evaluated in healthy adults during which the UES was augmented during the cough reflex $(18,19)$. However compared to the adult studies $(18,19)$, our population may exhibit lower UES protection as evidenced by lower UES pressure during cough, and/or lower pressure 
differential between the middle esophagus and UES (Table 1). This may lead to a decreased defense against microaspiration in BPD group who are already at high risk. (ii) It has been shown in normal adults that cough was followed by swallowing in only $52.9 \%$ (18), while in our study, cough was predominantly followed by swallowing in 90-92\% of cough episodes for spontaneous and stimulus respectively (Figure 2). This disparity may be maturation-related, or due to increased secretions or mucus present in infants with BPD. (iii) Adults with chronic cough have abnormal esophageal motility patterns, $85 \%$ of the time (16). We also noted abnormal esophageal motility patterns such as, nonpropagating swallow (59\%) and sphincter dysfunctions (69\%). (iv) In adults with proven GERD and chronic cough, the prevalence of abnormal esophageal motility ranged from 41 to $53 \%(16,17)$, whereas in our study, the coexistence of GER and abnormal esophageal motility was less evident but exists.

The cough reflex in newborn infants is not fully developed. It first becomes evident at $\sim 1-2$ mo age (3). On the other hand, cough equivalents may include prolonged exhalation, expiratory stridor, and the laryngeal chemoreflex. The laryngeal chemoreflex is an airway protective mechanism which involves obstructive apnea, swallowing, and/or laryngeal closure, the pharyngo-glottal closure reflex or the esophago-glottal closure reflex $(20,21)$. In premature infants, the laryngeal chemoreflex is more frequent and prolonged than in healthy, term infants (22). However, with advanced maturation, this reflex transitions into a cough response (3). Cough prevalence at 6-12 mo corrected age ranges from 50 to $80 \%$ in infants born prematurely $(23,24)$. In premature infants, we have previously evaluated the effects of physical and chemical stimuli on aerodigestive reflexes and the neural pathways likely involved (21,25-29). For example, pharyngeal stimulation activates a series of protective reflexes including pharyngeal reflexive swallowing, glottal closure, and deglutition apnea $(21,25,27)$. Risks for laryngeal penetration, aspiration, and coughing can occur if a pharyngeal stimulated reflexive swallow is nonpropagating or not associated with deglutition apnea. Thus, the occurrence of pharyngeal reflexive swallowing followed by exaggerated UES contractile reflex can hold back a retained bolus that has not been completely cleared. Given this scenario, the presence of UES contractility in the absence of prior complete bolus clearance can lead to increased risk for spill into the laryngeal vestibule and subsequent penetration or aspiration. However, activation of the pharyngo-UES contractile reflex (27) can also result in delayed pharyngeal clearance if not succeeded by UES relaxation as in swallowing. This scenario is also a prelude to bolus spill into the laryngeal vestibule, potentially leading to laryngeal penetration or aspiration. The pharyngo-glottal closure reflex may also be activated during this sequence (20), with cough resulting from the abrupt stimulation. Similarly, esophageal stimulation (as in GER events) may activate the UES-contractile reflex, secondary peristalsis, deglutition response, or glottal closure reflexes $(20,21,27,29,30)$. Susceptibility for aspiration increases with incomplete or inadequate glottal closure during swallowing or during GER events.
Weak defense mechanisms against retrograde aspiration from GER events may include a hypotonic UES, improper timing of the UES contractile reflex and/or low magnitude of the UES contractile reflex. Conversely, a weak defense mechanism against anterograde aspiration from oropharyngeal swallowing may include a hypertonic UES, improper timing of the UES relaxation reflex, and/or inadequate relaxation of the UES relaxation reflex. Similarly, cough can also have its origins from dysfunction at the level of the distal esophagus and LES. A hypotonic LES may cause an increased risk of reflux events. If proximal migration of the refluxate occurs, coughing may ensue. Conversely, if the LES exhibits incomplete relaxation, absence of relaxation, or presence of sustained contraction, esophageal peristaltic activity remains impaired because the bolus fails to clear effectively into the stomach.

Implications from our study may be applicable to the phenotype similar to that of our study population of a select group of infants with BPD, feeding difficulties and coughing. The frequency-occurrence of the characterized aerodigestive mechanisms of cough causation or resolution is therefore representative of such a phenotype. Clinical and research implications of this study are several: (i) The identification of initial aerodigestive mechanisms associated with cough and ameliorating mechanisms may assist in the recognition of appropriate therapeutic targets. (ii) The degree of cough reflex protection, the duration of these events, and the effects of respiratory rhythm disturbance on pulmonary function remain to be ascertained. (iii) Cough in the premature infant with BPD can occur by term postmenstrual age (PMA), as infants begin to be increasingly aware of their sensory perception, presenting as luminal clearance primarily with swallowing. (iv) Stimulus (type, duration of exposure, area of stimulation) presented can activate a cascade of reflexes, expectoration or swallowing, thoraco-abdominal dys-synchrony and potential changes in pulmonary functions. (v) Causes of cough in BPD infants with feeding difficulties can be several, and potential therapies can also be several, and may include oromotor and physical therapies to enhance/improve swallowing skills and improve UES function, suppressing airway secretions and inflammation. (vi) Abnormal modulation with proximal aerodigestive sensitivity and delays in clearance mechanisms are suggestive of deficits in central pattern generation and vagal neuropathy.

Like most clinical studies, our study had limitations. Our study subjects are a highly selected group of only 16 infants with severe BPD with feeding difficulties and coughing during manometric evaluation. The frequencies of occurrence of the various aerodigestive mechanisms related to cough and cough resolution are therefore representative of such a phenotype and not that of cough in the general population. Since studies were retrospectively selected based upon evidences of coughing during manometric evaluation, infants who did not cough during the study may have been excluded resulting in an underestimation of cough prevalence in this population. Thus, there was no control group to compare, since chronic cough is a manifestation of disease. Also, we have only highlighted the recognizable initial mechanisms of cough detected during 
manometry in this highly select population of BPD who may have multisystemic issues. However, there may be other respiratory causes of cough including but not limited to upper respiratory infections, pneumonia, excessive secretions, and inability to swallow secretions, or pulmonary inflammation which may involve airway hyper-reactivity, tracheitis, bronchiolitis, and atelectasis not detectable by manometry. There were some cough events not associated with aerodigestive events. It is likely that other factors are involved. We believe that the addition of impedance measurements may be helpful in determining direction of bolus movement to enhance detection of nonpropagating swallow and mechanisms of GER likely associated with cough episodes. Similarly, the addition of impedance would have allowed us to objectively determine if the post-tussive swallow terminated the cough or if it is just an association with the cough episode. There may be clinical usability despite limitations. For example, in those infants with lung disease who frequently experience coughing and pneumonias, providers commonly treat infants empirically with proton pump inhibitor therapies due to suspicion of GERD. However, in this pilot study, GER mechanisms were only associated in $20 \%$ of cough episodes. Therefore, our study approaches may be advantageous in defining the mechanisms of cough so as to narrow down the appropriate therapies with objective evidence.

In conclusion, esophageal dysmotility mechanisms were evident during and after cough. These mechanisms may be related to abnormalities with peristaltic clearance, heightened sensitivity, UES dysfunction, LES dysfunction, incoordination of central pattern generation, and/or gastroesophageal reflux. Evidence of multiple primary peristaltic events after cough may be a response to excessive secretions (generated by the vagal effects on mucus glands via muscarinic receptors), or mechano-sensitive and chemo-sensitive stimuli $(28,29,31)$. Primary peristalsis remains the most important clearance mechanism for post-tussive aerodigestive clearance a

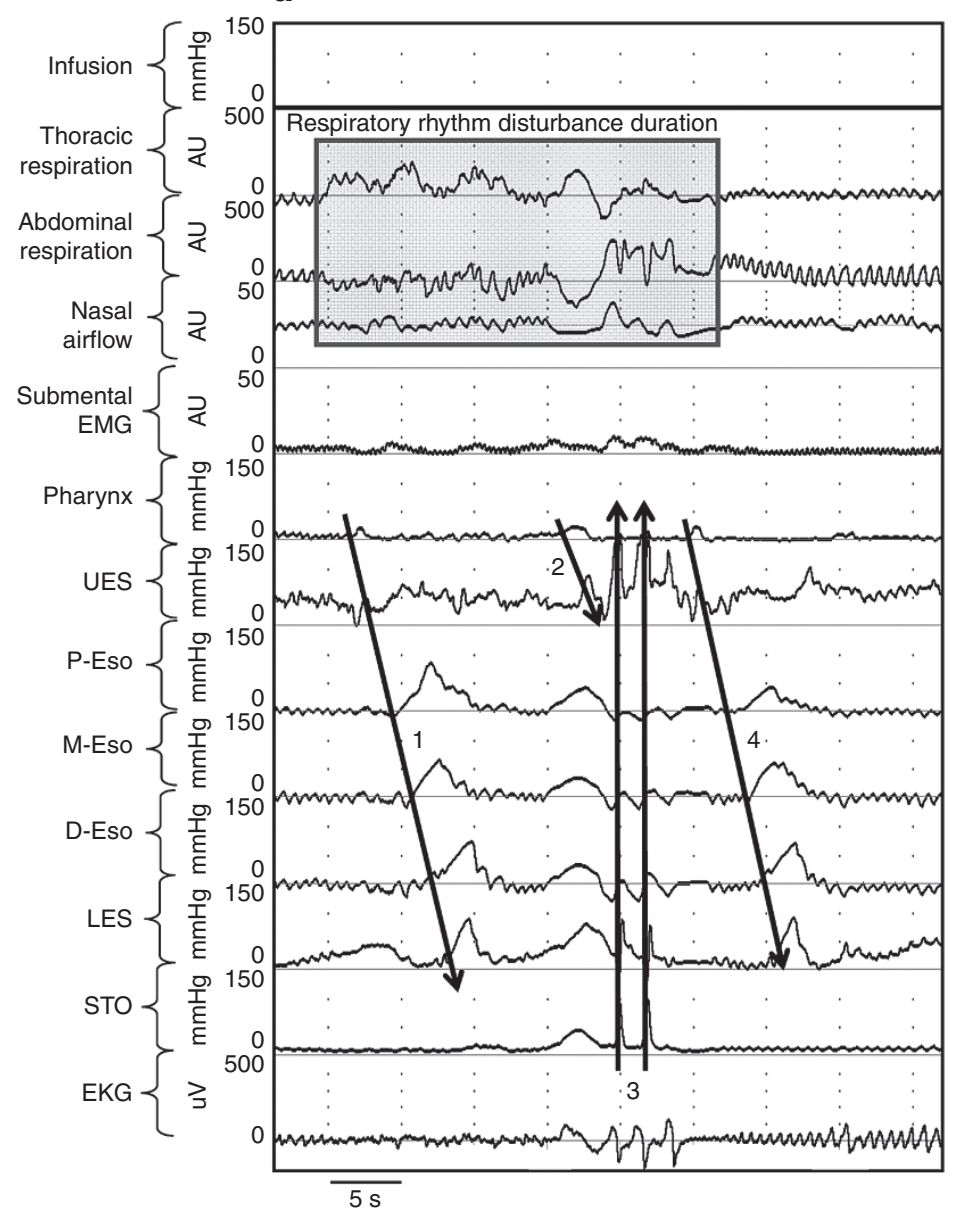

b

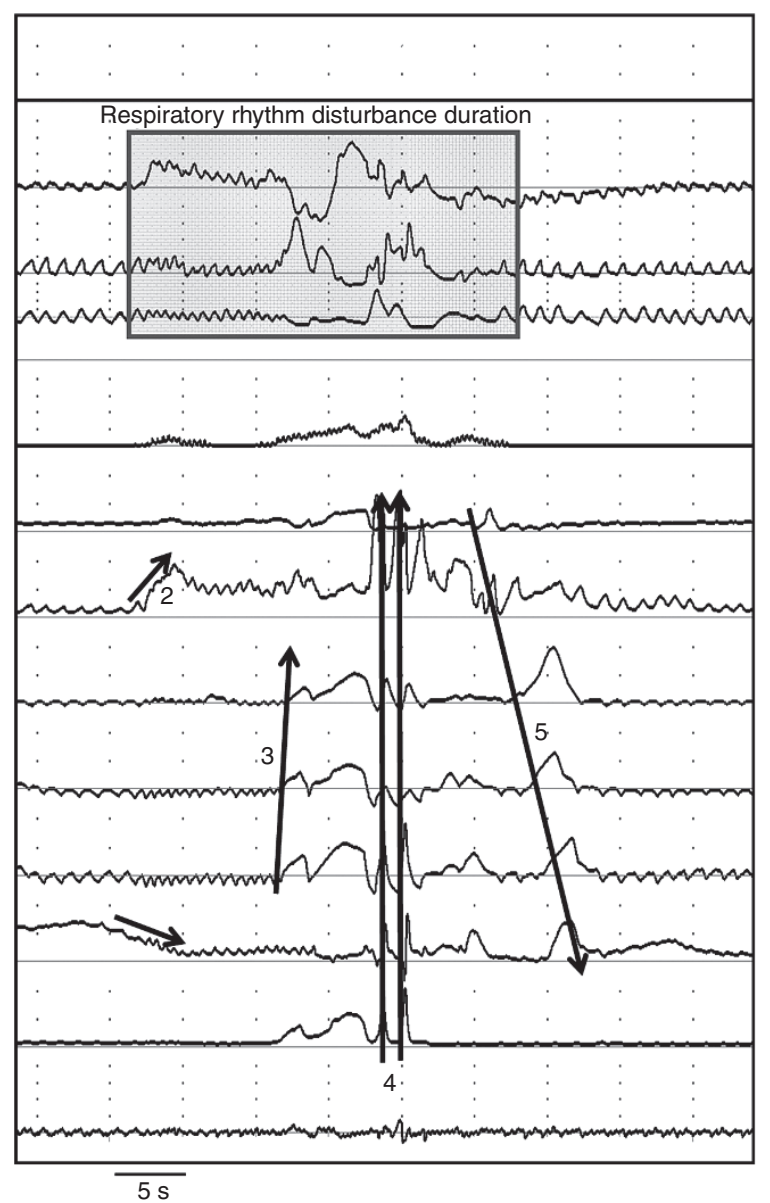

Figure 3. Spontaneous coughing associated with aerodigestive mechanisms: (a) Association with swallowing: Note the sudden 27.0 s disruption of breathing pattern including an episode of cessation of change in nasal airflow with associated respiratory rhythm disturbance. During this time there is (i) a fully propagated swallow followed by, (ii) nonpropagating swallow as the initial aerodigestive mechanism, (iii) multiple coughs, and (iv) complete primary peristalsis restoring aerodigestive and respiratory normalcy. (b) Association with reflux: Note the sudden $25.8 \mathrm{~s}$ disruption of breathing pattern including an episode of cessation of change in nasal airflow with associated respiratory rhythm disturbance. During this time, there is reflux as the initial aerodigestive mechanism characterized by (i) lower esophageal sphincter relaxation, (ii) upper esophageal sphincter contraction, (iii) common cavity (simultaneous pressure increase in the esophageal channels signifying esophageal retrograde movement) followed by (iv) multiple coughs, and (v) complete primary peristalsis restoring aerodigestive and respiratory normalcy 
a

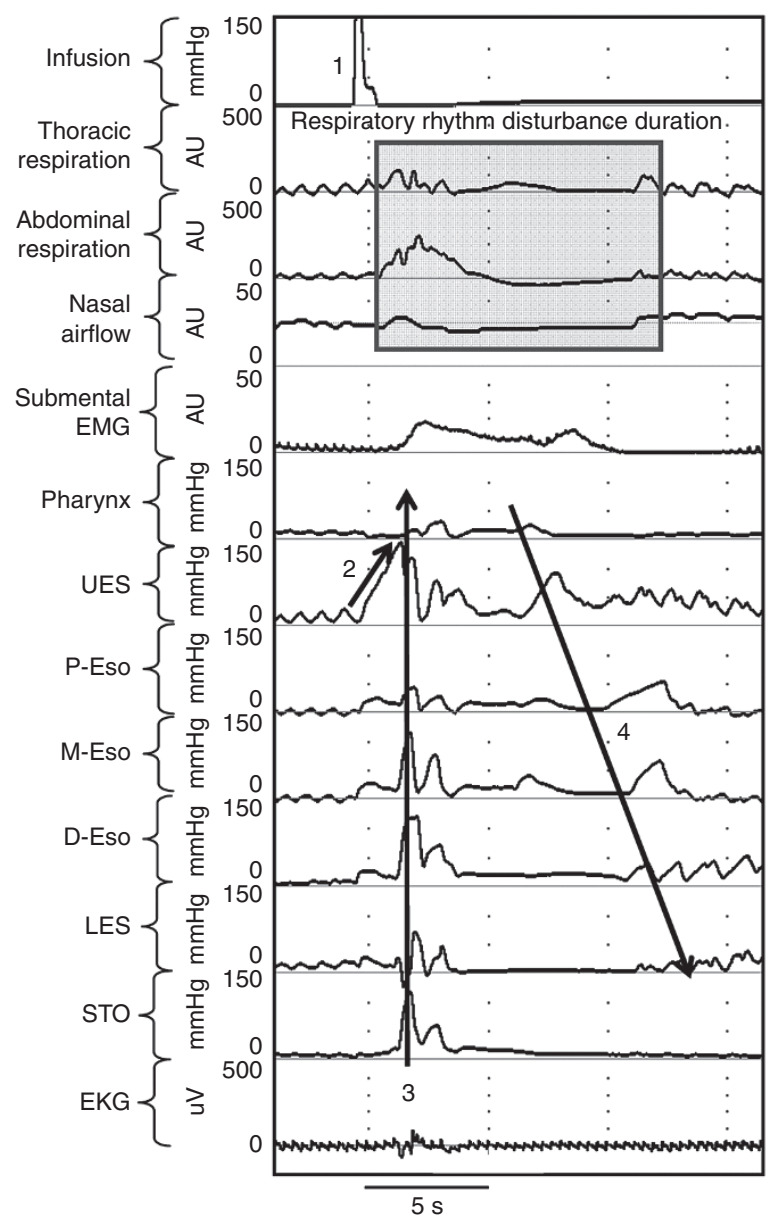

b

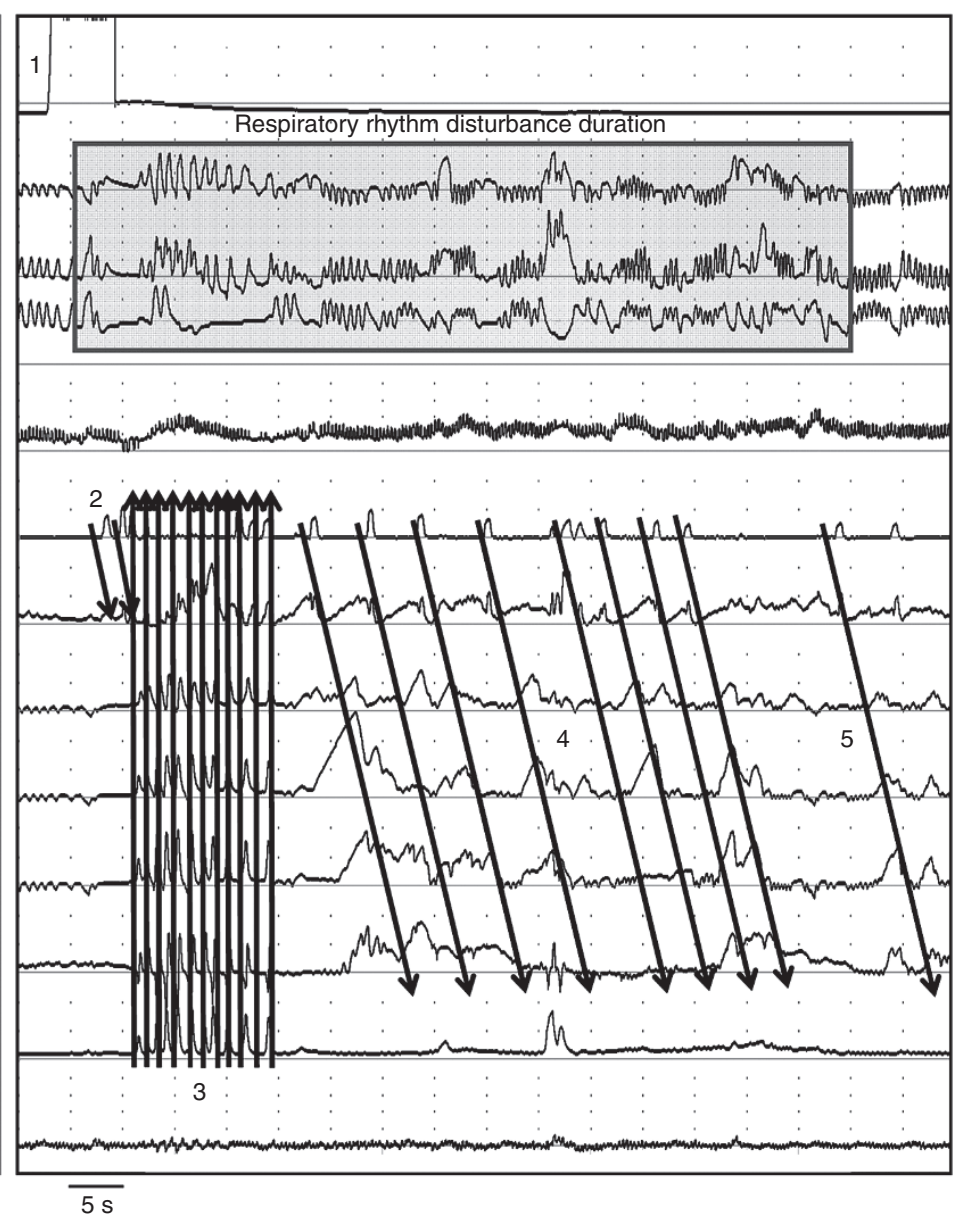

Figure 4. Stimulus induced coughing associated with aerodigestive mechanisms: (a) Cough reflex in response to esophageal stimulation: Note (i) $2 \mathrm{ml}$ air introduced into the mid-esophagus, (ii) associated with upper esophageal sphincter contractile reflex as the initial aerodigestive mechanism with respiratory rhythm disturbance $(10.8 \mathrm{~s}$ ) characterized by cessation or respiratory activity, (iii) cough, and (iv) complete primary peristalsis restoring aerodigestive and respiratory quiescence. (b) Cough reflex in response to pharyngeal stimulation: Note (i) $0.3 \mathrm{ml}$ water introduced into the pharynx, the respiratory rhythm disturbance (74.7 s) characterized by irregularly spaced bursts of rapid breathing efforts during which there is (ii) multiple failed swallowing attempts as the initial aerodigestive mechanism, and (iii) multiple cough events, (iv) attempts of aerodigestive restoration, and (v) complete peristalsis restoring aerodigestive and respiratory quiescence

and secondary peristalsis the most important following GER events. Premature infants with BPD and deglutitive impairment or with GER are at increased risk of consequences of cough and reactive airway disease. This may be due to immaturity of the sensory-motor aspects of aerodigestive reflexes.

\section{METHODS}

\section{Subjects}

Participants were selected from a larger study $(N=69)$ to evaluate esophagus-airway interactions of inpatient infants with BPD. Subject inclusion criteria were: (i) preterm-born infants that required ventilation prior to evaluation but not at time of evaluation, (ii) BPD at $36 \mathrm{wk}$ postmenstrual age per National Institutes of Health consensus criteria, (iii) enterally fed at evaluation, (iv) physiologically stable without any new onset illness at time of evaluation. Subject exclusion criteria were: (i) acid suppressive agents or narcotics at time of evaluation, and (ii) congenital anomalies. From this larger cohort, infants that exhibited coughing during the esophageal motility procedure were selected for analysis $(N=16)$. Infants analyzed were born from $11 / 2004$ to $6 / 2011$.

Study procedures and protocols were approved by the Institutional Review Board at The Research Institute at Nationwide Children's
Hospital, Columbus, Ohio. Signed informed parental consent and Health Insurance Portability and Accountability Act authorization were obtained prior to participation. Infants were evaluated in the neonatal intensive care unit setting at Nationwide Children's Hospital, Columbus, $\mathrm{OH}$. Modes of respiratory support (nasal continuous positive airway pressure, nasal cannula oxygen, or breathing room air) were not modified during evaluation as to not interfere with the subjects BPD management plan. All studies were done at the crib-side and closely monitored by a registered nurse and physician.

\section{Pharyngo-Esophageal Manometry Study Design}

Infants underwent evaluation of aerodigestive reflexes using basal and provocative esophageal manometry techniques as described and validated previously $(20,21,26-29,31-34)$. A custom designed size appropriate silicone pharyngo-esophageal manometry catheter (Dentsleeve International, Mui Scientific, Mississauga, Ontario, Canada) was comprised of the following: (i) 5 microlumen channels for pharynx, proximal-, middle- and distal- esophagus, and gastric contractions, (ii) dual sleeves for the UES and LES, (iii) and 2 microlumen infusion ports for pharyngeal and middle esophageal stimulation was to record pharyngo-esophageal waveform responses. All microlumen are manufactured into a single 6 FR catheter to be inserted transnasally. This catheter was connected to a micromanometric pharyngo-esophageal 
water perfusion system with amplifiers (Stationary Solar Gastro, v. 8.21 Medical Measurement Systems, Dover, NH), resistors (Dentsleeve International) with rates of $0.01,0.02,0.04$, and $0.08 \mathrm{ml} / \mathrm{min}$ dependent upon subject size, and pressure transducers (Ohmeda TNF-R disposable transducers; Medical Measurement Systems) to record waveform changes during basal and adaptive states. Prior to placement, catheter was zeroed at the level of the subjects esophagus and verified at study end. Catheter was placed nasally by a trained physician in the supine lying infant and appropriately positioned using the pull through technique and $15 \mathrm{~min}$ time allotted for infant adaptation before stimulation was carried out. Standard protocol includes graded volumes of esophageal (air, sterile water, or apple juice at volumes of $0.1,0.5,1,2$, or $5 \mathrm{ml}$ in triplicate) and pharyngeal stimulation (sterile water at volumes of $0.1,0.3,0.5$ in triplicate) through the infusion ports by the physician to test aerodigestive reflexes $(20,21,26-29,31)$. To achieve the aims of the current study, cough clusters were evaluated and categorized as spontaneous (no stimulus administered and associated with cough) or stimulus induced (pharyngeal or esophageal stimulus administered and associated with cough).

\section{Physiological Measurement Study Design}

Respiratory and airflow recordings were acquired concurrently with pharyngo-esophageal manometry to determine associated respiratory changes and temporal relationships with esophageal motility (27-29,31,35-38). Respiratory waveforms were documented using respiratory inductance plethysmography (Respitrace, Viasys, Conshohocken, PA) to detect and record thoracic rib cage and abdominal wall movements via secure elastic bands with inductance coils (Respibands, CareFusion, Minneapolis, MN). During inspiration, thoraco-abdominal cross sectional area increases, resulting in waveform upstroke. Conversely, during expiration thoraco-abdominal cross sectional area decreases, resulting in waveform down-stroke. Direction of air flow was monitored using a nasal thermistor (Integra Life Sciences, Plainsboro, NJ) to detect thermal differences between inhaled and exhaled air. Ambient air temperature is cooler than body temperature. Therefore, during inspiration the atmospheric air is warmed through the naso-pharynx and airway resulting in a higher temperature of air flowing through lungs and back out during expiration. Hence, study convention was to set thermistor polarity so that waveform upstroke corresponded to inspiration (cooler temperature) and waveform down-stroke corresponded to expiration (warmer temperature).

\section{Definitions}

Cough events are individual occurrences detected along with the combinations: (i) an audible cough sound, manually marked at time of occurrence, (ii) markers of respiratory inductance plethysmographic changes demonstrating a deep inhalation followed by deep exhalation, (iii) airflow cessation on nasal thermistor, and (iv) an abrupt intra-thoracic simultaneous pressure increase in the esophageal channels and increase in gastric pressure (a signature of cough in manometry) (19). Cough clusters were defined as the occurrence of either a solitary cough event or multiple cough events in rapid succession. Stimulus induced cough clusters were defined as cough clusters occurring within $20 \mathrm{~s}$ following esophageal or pharyngeal stimulation. Spontaneous cough clusters were defined as cough clusters occurring separate from any esophageal or pharyngeal stimulation.

Respiratory rhythm disturbance was defined as an interruption in the regular sinusoidal breathing pattern lasting longer than $2 \mathrm{~s}$ as assessed from respiratory inductance plethysmography and nasal airflow thermistor. Duration was calculated as the period between the onset and end of this disturbance. Potential aerodigestive esophageal reflex mechanisms associated with cough included UES contractile reflex, LES relaxation, esophageal retrograde movement, nonpropagating swallow, or none identifiable (cough only). UES contractile reflex was defined as an increase in $4 \mathrm{mmHg}$ above basal UES pressure $(32,38)$. LES relaxation was defined as a drop in of at least $5 \mathrm{mmHg}$ below basal LES pressure (32). Refluxogenic mechanisms, such as transient lower esophageal sphincter relaxation and esophageal retrograde movement, were distinguished by esophageal manometry patterns. Nonpropagating swallow was determined by occurrence of incomplete or failed peristalsis. The initial aerodigestive mechanism associated with cough was defined as the first esophageal event in time that occurred before the onset of the coughing cluster. Since multiple aerodigestive mechanisms could be associated with cough after the initial mechanism, subsequent aerodigestive event patterns were also identified.

The post-tussive clearance mechanism was defined as the final esophageal event in time that restored respiratory and esophageal quiescence within the aerodigestive tract. Potential esophageal clearance mechanisms were primary peristalsis, secondary peristalsis, or none identifiable $(28,29,31)$. Both primary and secondary peristalsis require esophageal body contraction and LES relaxation, however primary peristalsis exhibits pharyngeal contractile waveform with UES relaxation, while secondary peristalsis exhibits absence of pharyngeal activity with UES contraction $(28,29,31)$. Clearance mechanisms were also analyzed for propagation characteristics (complete, incomplete, or failed). Complete propagation is described as sequential anterograde movement of esophageal waveforms, progressing from the proximal esophagus to the distal esophagus. Incomplete peristalsis is defined as two or fewer waveforms reaching the distal esophagus. Failed peristalsis is defined as the absence of esophageal waveforms despite a pharyngeal peak and UES relaxation (34).

\section{Analysis of Physiologic Recordings}

Relationships between respiratory waveforms and aerodigestive reflex changes during cough events were analyzed by evaluating esophageal topography at the manometric domains of UES, mid-esophagus, LES and stomach befo re, during, and after cough clusters. Manometric changes were recorded during quiescence before and after the cough cluster to determine pre- and post- tussive baseline measurements. Post-tussive quiescence was described as the return to baseline of respiratory rhythm and esophageal waveform normalcy and esophageal mechanisms that restored these were assessed. Cough onset, peak, and offset pressures $(\mathrm{mmHg})$ were recorded based on gastric contractile waveforms.

Initial aerodigestive mechanisms associated with cough were subgrouped relative to aerodigestive tract origin: (i) upper aerodigestive (UES contractile reflex, nonpropagating swallow), (ii) lower aerodigestive (LES relaxation, esophageal retrograde movement), and (iii) airway (cough only event). Since there are multiple other causes of cough not related to the the digestive tract (including but not limited to upper respiratory tract infections, pneumonia, excessive secretions, hyper-reactivity or those specific to the BPD population with pulmonary inflammation which may involve tracheitis, bronchiolitis, and atelectasis), the airway subgroup would potentially include these cases. A representative example of initial aerodigestive mechanisms associated with cough and clearance mechanisms can be observed for both spontaneous and stimulus induced clusters (Figures 3 and 4).

The potential influence of current levels of respiratory support (NCPAP vs. nasal cannula oxygen or room air (non-NCPAP)) on initial aerodigestive mechanisms associated with cough and clearing mechanisms were examined.

\section{Statistical Analysis}

Statistical analysis was performed using SigmaPlot 12.0 (Stystat Software, San Jose, CA). Shapiro-Wilk tests were used to determine normality. Since pressure values were not normally distributed, comparisons were made using Kruskal-Wallis one-way analysis of variance on rank (Table 1). Tukey tests and Dunn's methods were used for all pairwise multiple comparison procedures. $\chi^{2}$ was used for analysis and was also used for comparison between the groups. Multinomial logistic regression was used to generate odds ratios. $P$ values $<0.05$ were considered statistically significant. Values are presented as mean $\pm \mathrm{SE}$, median (range), or $n(\%)$.

\section{ACKNOWLEDGMENTS}

The authors would like to acknowledge Rebecca Moore, RN, BSN, and Brittany Durr, RN, BSN, MHA for providing nursing support.

\section{STATEMENT OF FINANCIAL SUPPORT}

This study was supported by National Institutes of Health, Bethesda, MD, P01 DK 068051 (Jadcherla/Shaker).

Disclosure: The authors have no real or perceived conflicts of interest to declare. 


\section{REFERENCES}

1. Shaker R. Reflex interaction of pharynx, esophagus, and airways. GI Motility Online [online] 2006. http://www.nature.com/gimo/contents/pt1/full/ gimo11.html doi:10.1038/gimo11.

2. Hadjikoutis S, Wiles CM, Eccles R. Cough in motor neuron disease: a review of mechanisms. QJM 1999;92:487-94.

3. Thach BT. Maturation of cough and other reflexes that protect the fetal and neonatal airway. Pulm Pharmacol Ther 2007;20:365-70.

4. Canning BJ. Anatomy and neurophysiology of the cough reflex: ACCP evidence-based clinical practice guidelines. Chest 2006;129(1 Suppl):33S-47S.

5. Irwin RS, Baumann MH, Bolser DC, et al. Diagnosis and management of cough executive summary: ACCP evidence-based clinical practice guidelines. Chest 2006;129:1S-23S.

6. Chan CY, Jadcherla SR. Relationship between cough, irritability and arching with gastroesophageal reflux (GER) in premature infants with chronic lung disease (CLD). Gastroenterology 2012;142: S-48.

7. Jobe AH, Bancalari E. Bronchopulmonary dysplasia. Am J Respir Crit Care Med 2001;163:1723-9.

8. Stevenson DK, Wright LL, Lemons JA, et al. Very low birth weight outcomes of the National Institute of Child Health and Human Development Neonatal Research Network, January 1993 through December 1994. Am J Obstet Gynecol 1998;179(6 Pt 1):1632-9.

9. Stoll BJ, Hansen NI, Bell EF, et al.; Eunice Kennedy Shriver National Institute of Child Health and Human Development Neonatal Research Network. Neonatal outcomes of extremely preterm infants from the NICHD Neonatal Research Network. Pediatrics 2010;126:443-56.

10. Ehrenkranz RA, Walsh MC, Vohr BR, et al.; National Institutes of Child Health and Human Development Neonatal Research Network. Validation of the National Institutes of Health consensus definition of bronchopulmonary dysplasia. Pediatrics 2005;116:1353-60.

11. Johnson TJ, Patel AL, Jegier BJ, Engstrom JL, Meier PP. Cost of morbidities in very low birth weight infants. J Pediatr 2013;162:243-49.e1.

12. Mizuno K, Nishida Y, Taki M, et al. Infants with bronchopulmonary dysplasia suckle with weak pressures to maintain breathing during feeding. Pediatrics 2007;120:e1035-42.

13. Gewolb IH, Vice FL. Abnormalities in the coordination of respiration and swallow in preterm infants with bronchopulmonary dysplasia. Dev Med Child Neurol 2006;48:595-9.

14. Mercado-Deane MG, Burton EM, Harlow SA, et al. Swallowing dysfunction in infants less than 1 year of age. Pediatr Radiol 2001;31:423-8.

15. McGrath-Morrow SA, Hayashi M, Aherrera AD, Collaco JM. Respiratory outcomes of children with BPD and gastrostomy tubes during the first 2 years of life. Pediatr Pulmonol 2014;49:537-43.

16. Kastelik JA, Redington AE, Aziz I, et al. Abnormal oesophageal motility in patients with chronic cough. Thorax 2003;58:699-702.

17. Fouad YM, Katz PO, Hatlebakk JG, Castell DO. Ineffective esophageal motility: the most common motility abnormality in patients with GERDassociated respiratory symptoms. Am J Gastroenterol 1999;94:1464-7.

18. Xiao Y, Carson D, Boris L, et al. The acoustic cough monitoring and manometric profile of cough and throat clearing. Dis Esophagus 2014;27:5-12.

19. Amaris M, Dua KS, Naini SR, Samuel E, Shaker R. Characterization of the upper esophageal sphincter response during cough. Chest 2012;142:122936.

20. Jadcherla SR, Gupta A, Wang M, Coley BD, Fernandez S, Shaker R. Definition and implications of novel pharyngo-glottal reflex in human infants using concurrent manometry ultrasonography. Am J Gastroenterol 2009;104:2572-82.
21. Jadcherla SR, Gupta A, Coley BD, Fernandez S, Shaker R. Esophago-glottal closure reflex in human infants: a novel reflex elicited with concurrent manometry and ultrasonography. Am J Gastroenterol 2007;102:2286-93.

22. Pickens DL, Schefft GL, Thach BT. Pharyngeal fluid clearance and aspiration preventive mechanisms in sleeping infants. J Appl Physiol (1985) 1989;66:1164-71.

23. Greenough A, Limb E, Marston L, Marlow N, Calvert S, Peacock J. Risk factors for respiratory morbidity in infancy after very premature birth. Arch Dis Child Fetal Neonatal Ed 2005;90:F320-3.

24. Pramana IA, Latzin P, Schlapbach LJ, et al. Respiratory symptoms in preterm infants: burden of disease in the first year of life. Eur J Med Res 2011;16:223-30.

25. Jadcherla SR, Gupta A, Stoner E, Coley BD, Wiet GJ, Shaker R. Correlation of glottal closure using concurrent ultrasonography and nasolaryngoscopy in children: a novel approach to evaluate glottal status. Dysphagia 2006;21:75-81.

26. Jadcherla SR, Shubert TR, Gulati IK, Jensen PS, Wei L, Shaker R. Upper and lower esophageal sphincter kinetics are modified during maturation: effect of pharyngeal stimulus in premature infants. Pediatr Res 2015;77:99-106.

27. Jadcherla SR, Gupta A, Stoner E, Fernandez S, Shaker R. Pharyngeal swallowing: defining pharyngeal and upper esophageal sphincter relationships in human neonates. J Pediatr 2007;151:597-603.

28. Jadcherla SR, Hoffmann RG, Shaker R. Effect of maturation of the magnitude of mechanosensitive and chemosensitive reflexes in the premature human esophagus. J Pediatr 2006;149:77-82.

29. Gupta A, Gulati P, Kim W, Fernandez S, Shaker R, Jadcherla SR. Effect of postnatal maturation on the mechanisms of esophageal propulsion in preterm human neonates: primary and secondary peristalsis. Am J Gastroenterol 2009;104:411-9.

30. Jadcherla SR. Pathophysiology of aerodigestive pulmonary disorders in the neonate. Clin Perinatol 2012;39:639-54.

31. Jadcherla SR. Manometric evaluation of esophageal-protective reflexes in infants and children. Am J Med 2003;115 Suppl 3A:157S-60S.

32. Pena EM, Parks VN, Peng J, et al. Lower esophageal sphincter relaxation reflex kinetics: effects of peristaltic reflexes and maturation in human premature neonates. Am J Physiol Gastrointest Liver Physiol 2010;299: G1386-95.

33. Jadcherla SR, Duong HQ, Hofmann C, Hoffmann R, Shaker R. Characteristics of upper oesophageal sphincter and oesophageal body during maturation in healthy human neonates compared with adults. Neurogastroenterol Motil 2005;17:663-70.

34. Jadcherla SR, Parks VN, Peng J, et al. Esophageal sensation in premature human neonates: temporal relationships and implications of aerodigestive reflexes and electrocortical arousals. Am J Physiol Gastrointest Liver Physiol 2012;302:G134-44.

35. Hodsman NB, Kenny GN, Rennie R, McArdle CS. Data collection and analysis from a respiratory inductance plethysmograph. Int J Clin Monit Comput 1987;4:237-41.

36. Reber A, Geiduschek JM, Bobbià SA, Bruppacher HR, Frei FJ. Effect of continuous positive airway pressure on the measurement of thoracoabdominal asynchrony and minute ventilation in children anesthetized with sevoflurane and nitrous oxide. Chest 2002;122:473-8.

37. Hammer J, Newth CJ. Assessment of thoraco-abdominal asynchrony. Paediatr Respir Rev 2009;10:75-80.

38. Jadcherla SR, Duong HQ, Hoffmann RG, Shaker R. Esophageal body and upper esophageal sphincter motor responses to esophageal provocation during maturation in preterm newborns. J Pediatr 2003;143:31-8. 